\title{
Significant effects of implementation of health-promoting schools on schoolteachers' nutrition knowledge and dietary intake in Taiwan
}

\author{
Yi-Hua Chen ${ }^{1}$, Ching-Ying Yeh ${ }^{1}$, Yu-Ming Lai ${ }^{1}$, Meei-Ling Shyu ${ }^{2}$, Kuo-Cherh Huang ${ }^{3}$ and \\ Hung-Yi Chiou, * \\ ${ }^{1}$ School of Public Health, College of Public Health, Taipei Medical University, No. 250 Wu-hsing Street, \\ Taipei 1 10, Taiwan: ${ }^{2}$ School of Nursing, College of Nursing, Taipei Medical University, Taipei, Taiwan: \\ ${ }^{3}$ School of Health Care Administration, Taipei Medical University, Taipei, Taiwan
}

Submitted 14 April 2008: Accepted 25 June 2009: First published online 6 August 2009

\begin{abstract}
Objective: Although teachers are the key participants in health-promoting schools (HPS) programme delivery, it is still unknown whether teachers are appropriate health information resources and role models for students with respect to healthy diets. The present study aimed to investigate the effects of implementing HPS programmes on teachers' nutrition knowledge and diets.

Design: One HPS programme aiming at dietary intervention (HP-D) and one HPS not aiming at dietary intervention (HP-ND) were selected, along with two nonhealth-promoting (NHP) schools matched for school size and urbanization level with the two HPS. All 361 teachers in the four schools were invited to participate, yielding a 78.4\% overall valid response rate. A structured, self-reported questionnaire was administered, with regression models used for statistical analysis. Results: Teachers in the HP-D group had a mean score of $21 \cdot 1$ on a range of $0-30$ for nutrition knowledge, which was significantly higher than the mean scores of $18 \cdot 5$ in the HP-ND group and $19 \cdot 1$ in the NHP group $(P<0 \cdot 001)$. Better dietary behaviours were also observed among HP-D teachers. Further, being a 'health education' course instructor was associated with significantly higher scores on nutrition knowledge $(\beta=2 \cdot 6, P<0 \cdot 001)$ and vegetable and fruit consumption $(\beta=1 \cdot 4, P=0 \cdot 02)$ in the HP-D group than in the NHP group. The HP-ND and NHP groups exhibited similar patterns of non-significant differences compared with the HP-D group.

Conclusions: Implementation of a coordinated HPS framework on nutrition and diet was positively correlated with schoolteachers' nutrition knowledge and dietary intake.
\end{abstract}

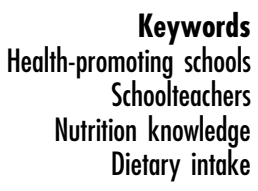

Inadequate diet and nutrition can slow growth in childhood and increase the risk of health conditions such as obesity, hypertension and certain cancers in adult$\operatorname{hood}^{(1,2)}$. Many children exhibit intakes of food and nutrients below the recommended levels, both internationally and in Taiwan ${ }^{(3-7)}$. Educators have recognized the importance of promoting a healthy diet and various nutrition-education programmes have been implemented in schools ${ }^{(8-10)}$.

In these school-based programmes, teachers play a central role in providing accurate nutrition information to students and responding to students' concerns about diet, nutrition and weight control. In order for schoolteachers to be a valuable source of health information, however, they must be equipped with adequate knowledge ${ }^{(11)}$. Teachers' knowledge of nutrition affects their willingness and confidence to teach a course on healthy diets ${ }^{(12)}$, which could influence the implementation of a nutritioneducation programme in their schools. In addition, teachers' unhealthy eating behaviours at school may make them inappropriate role models for students. In a study reported by Kubik et al. ${ }^{(13)}$, $62 \%$ of teachers reported purchasing beverages from vending machines. In addition, 54\% of teachers did not consume five servings of fruits and vegetables daily. Among physical education teachers, 20\% of women and $13 \%$ of men regularly skipped breakfast and $14 \%$ of women reported having an ongoing eating disorder. Potentially dangerous methods of weight loss were employed by teachers, with $19 \%$ of women abusing laxatives and $10 \%$ inducing vomiting ${ }^{(14)}$. It has also been suggested that junior teachers are likely influenced by the same psychosocial factors that affect body image in other 
young adults. Thus, teachers may transfer inappropriate attitudes or behaviours to their students ${ }^{(14)}$.

The health-promoting schools (HPS) framework was proposed to reflect the observation that most traditional health education aimed at individual behaviours had minimal and short-term effects ${ }^{(15,16)}$ and was relatively ineffective ${ }^{(15,17)}$. Thus, in the 1980s, the WHO shifted the focus of health education to the development of healthy 'settings' for school health promotion defined in the Ottawa Charter ${ }^{(18)}$. Implementation of the HPS framework offers an opportunity to incorporate health policy practices, curriculum administration and parent and community networks, with a concomitant emphasis on students, teachers and community members. Over the past decade, the HPS framework has been implemented globally. In Taiwan, a programme coordinated jointly by the Department of Health and the Ministry of Education was initiated in 2003, with six major elements in line with the WHO guidelines. Each participating school could choose additional special intervention programmes to incorporate into its HPS implementation. Over the years, about half of the schools in Taiwan have chosen 'nutrition and diet' as their special intervention, indicating general recognition of the relationship between malnutrition and diminished learning capacity, school aptitude and health conditions $^{(19,20)}$.

There is very limited published research focusing on the impact of HPS implementation on teachers' nutrition knowledge and dietary habits. It is not known whether teachers are appropriate health information resources and role models for students in terms of dietary knowledge and behaviours. This issue merits attention because teachers are the key participants in HPS programme delivery. It has been suggested that teachers may unintentionally do more harm than good in attempting to guide vulnerable students towards healthy eating behaviours and weight control ${ }^{(14)}$. Thus, it is important for teachers to equip themselves with accurate nutrition knowledge and proper dietary habits so that they can be effective nutrition educators and appropriate role models. Moreover, educators' nutrition knowledge and diet are closely related to their own dietary habits and health condition. Whether HPS implementation can simultaneously promote schoolteachers' health knowledge and behaviours should be further elucidated.

To address this, we explore the impact of HPS implementation on teachers' nutrition knowledge and behaviours. In order to distinguish between the effects of HPS that focused on 'nutrition and diet' and those that focused on other special themes, schools were classified into three types: (i) HPS aiming at dietary intervention (HP-D); (ii) HPS not aiming at dietary intervention (HP-ND); and (iii) non-health-promoting (NHP) schools. We examined the association between the effects of HPS status on schoolteachers' nutrition knowledge and dietary intake by weight-related status and teaching-related status.

\section{Methods}

\section{Subjects}

In 2005, twenty-five HPS were designated 'paradigms' from a total of 318 HPS in Taiwan. These paradigmatic schools were supported with extra resources for their selected special intervention programmes such as oral health and nutrition and diet. Of these twenty-five schools, only one elementary school selected the same theme of 'nutrition and diet' for two consecutive years; in addition, only one elementary school did not choose to intervene through 'nutrition and diet' in the same two consecutive years of 2005 and 2006. Considering that it would probably take longer than a year for an intervention programme to reveal its effects, these two schools were selected for the present study in January 2007 and designated the HP-D and HP-ND groups, respectively. Two NHP schools matched for school size and urbanization level with the HP-D and HP-ND schools were selected as controls. All schoolteachers in the HP-D, HPND and NHP schools were recruited to assess the effects of the dietary intervention in HPS.

\section{Procedures}

All teachers were requested to fill out a self-reported questionnaire individually together with an informed consent and return it to the main supervisor within a week. There were 283 valid questionnaires obtained from a total of 361 teachers in these four schools, for a $78.4 \%$ overall valid response rate.

\section{Instrument}

A structured $15 \mathrm{~min}$ self-reported questionnaire entitled 'Investigation on Nutrition Knowledge and Dietary Intake among Schoolteachers' was developed based on a thorough review of the available literature as well as interviews with public health and nutrition experts and practitioners. Seventy multiple-choice questions were distributed over four sections.

Part I assessed nutrition knowledge with five trueor-false questions in each of six categories: food classification; nutrient functions; weight control; food safety; recommended daily consumption in childhood; and results from a 2001 national study on schoolchildren's dietary intake. The responses were then coded, with 1 indicating 'correct' and 0 indicating 'incorrect' answers. Correct responses were then summed to generate a total nutrition knowledge score and six scores for each of the six categories.

Part II consisted of a series of questions on dietary behaviours in the past $30 \mathrm{~d}$, including six items of nutrient intake behaviours (e.g. being concerned about calories while eating and label reading for nutrient content when purchasing food) and three extra questions about eating breakfast every day, drinking water only and having five servings of vegetables and fruits daily ('five-a-day'). 
These were assessed with a 5-point Likert scale, with 1 indicating 'almost none' and 5 indicating 'always'. Six items regarding nutrient intake behaviours were summed into a total, with higher scores indicating a higher frequency of healthier behaviours.

Part III covered seventeen questions on the frequencies of consumption of various types of food in the preceding $7 \mathrm{~d}$, such as meat, vegetables, fruits, snacks and sweetened beverages. A 5-point Likert scale similar to that described above was applied, with 1 indicating 'never' and 5 indicating 'almost every day'. Responses in the same food category were summed (e.g. categorized as fatty food such as fried chicken, snacks and sweetened beverages, and vegetables and fruits), with higher scores indicating higher consumption frequencies.

Finally, all respondents were asked to report their height, weight and demographic information (sex, age and marital status). BMI was calculated as body weight divided by the square of height $\left(\mathrm{kg} / \mathrm{m}^{2}\right)$. Weight perception was characterized by the respondent's self-perception of being an appropriate weight or overweight. As it was reported by Wardle et al. that weight control attempts were frequent among Asian women with generally low body weights ${ }^{(21)}$, not only BMI but also weight perception would affect dietary habits. Thus, responses on BMI and weight perception were combined and recoded as 'BMI $<24 \mathrm{~kg} / \mathrm{m}^{2}$ and consider self to be an appropriate weight', 'BMI $<24$ $\mathrm{kg} / \mathrm{m}^{2}$ and consider self to be overweight' and 'BMI $\geq 24$ $\mathrm{kg} / \mathrm{m}^{2}$. This division was based on the criteria put forth by the Department of Health, Executive Yuan in Taiwan. Teachers' satisfaction with their overall physical fitness was measured and coded as 'satisfied' or 'unsatisfied'. Finally, respondents' teaching-related status was assessed, including how long they had taught and whether they were an in-class tutor or a 'health education' course instructor. In Taiwan, an in-class tutor is assigned for each class to be fully in charge of students' everyday affairs, including having lunch with students. Teachers' participation in nutritionrelated training in the past 3 years was also recorded.

Content validity was assessed and ascertained by six experts in public health and nutrition-related fields. Pilot studies were conducted in advance, and Cronbach's $\alpha$ for internal consistency ranged from $0 \cdot 64$ to $0 \cdot 80$.

\section{Data analysis}

The $\chi^{2}$ test of significance was used to identify differences in sociodemographic characteristics and weight- and teaching-related status by HPS status. One-way ANOVA was applied to examine the distributions of nutrition knowledge, dietary behaviours and frequencies of food consumption in the HP-D, HP-ND and NHP groups, with post hoc analysis of Scheffé multiple comparisons procedures administered for significant results with $F$ tests. Multivariate linear regression was used to calculate the regression coefficient $(\beta)$ of each dependent variable of dietary outcomes separately for HP-D compared with
NHP and for HP-ND compared with NHP. Models were stratified by BMI and weight perception, by physical fitness satisfaction and by each of the teaching-related status items and adjusted for sex, age and marital status. Finally, radar graphs were drawn for the examination of scores on six categories of nutrition knowledge and four particular dietary behaviours by HPS status. Statistical analyses were performed with the STATA statistical software package version $7 \cdot 0$ (Stata Corporation, College Station, TX, USA). All tests of significance were two-tailed, with the level of significance set at $P<0 \cdot 05$.

\section{Results}

\section{Characteristics of the sample}

The distribution of sociodemographic characteristics, weight-related status and teaching-related status by HPS status is displayed in Table 1. Approximately threequarters of the participants were female, representing a disproportionate sex ratio among schoolteachers in elementary schools. In addition, 38.9\% of HP-D schoolteachers had participated in nutrition-related training in the past 3 years, compared with only $12.7 \%$ in the HP-ND group and $18 \cdot 2 \%$ in the NHP group $(P<0 \cdot 001)$.

\section{Nutrition knowledge and dietary intake by bealtb- promoting scbool status}

Table 1 also displays the results for nutrition knowledge and dietary intake by HPS status. Teachers in the HP-D group obtained a mean score of $21 \cdot 1$ on a range of $0-30$ for nutrition knowledge, significantly higher than the mean scores of 18.5 in the HP-ND group and $19 \cdot 1$ in the NHP group $(P<0 \cdot 001)$. As shown in Fig. 1(a), scores for the six knowledge categories were approximately equally distributed among HP-D teachers, while those of HP-ND and NHP teachers revealed lower scores in the knowledge of food classification than in the remaining five subtypes. Teachers in the HP-D group had significantly higher scores than those in the HP-ND and NHP groups in three of the knowledge categories.

For dietary intake behaviours, it was observed that HP-D schoolteachers had better nutrient intake behaviours than teachers in the other two school categories, while HP-ND teachers had the highest frequencies of fatty food consumption (Table 1). Regarding particular dietary behaviours, it was found that a significantly higher proportion ( $88.9 \%)$ of HP-D teachers 'always' eat breakfast compared with those in the HP-ND (73.6\%) and NHP $(76 \cdot 3 \%)$ groups $(P=0 \cdot 048)$. Significantly higher proportions of HP-D teachers 'always' consumed five servings of vegetables and fruits daily and read labels for nutrient content when purchasing food $(19 \cdot 4 \%$ and $32 \cdot 4 \%$, respectively) compared with those in the HP-ND $(5 \cdot 6 \%$ and $20 \cdot 8 \%$, respectively) and NHP (10.8\% and $15 \cdot 8 \%$, respectively) groups (Fig. 1(b)). 
Table 1 Distributions of sociodemographic characteristics, weight-related status, teaching-related status, nutrition knowledge and dietary intake by health-promoting school status (Taiwan, 2007)

\begin{tabular}{|c|c|c|c|c|c|c|c|}
\hline & \multicolumn{6}{|c|}{ Health-promoting school status } & \multirow[b]{3}{*}{$P$ value $\ddagger$} \\
\hline & \multicolumn{2}{|c|}{ HP-D } & \multicolumn{2}{|c|}{ HP-ND } & \multicolumn{2}{|c|}{ NHP } & \\
\hline & $n+$ & $\%$ & $n+$ & $\%$ & $n+$ & $\%$ & \\
\hline \multicolumn{8}{|l|}{ A. Sociodemographic characteristics } \\
\hline & & & & & & & $0 \cdot 341$ \\
\hline Male & 13 & $18 \cdot 1$ & 19 & $26 \cdot 8$ & 37 & $26 \cdot 6$ & \\
\hline Female & 59 & $81 \cdot 9$ & 52 & $73 \cdot 2$ & 102 & $73 \cdot 4$ & \\
\hline Age (years) & & & & & & & $<0.001$ \\
\hline $20-29$ & 15 & $22 \cdot 7$ & 39 & $60 \cdot 0$ & 25 & $19 \cdot 4$ & \\
\hline 30-39 & 33 & $50 \cdot 0$ & 18 & $27 \cdot 7$ & 64 & $49 \cdot 6$ & \\
\hline $40+$ & 18 & $27 \cdot 3$ & 8 & $12 \cdot 3$ & 40 & $31 \cdot 0$ & \\
\hline Marital status & & & & & & & 0.002 \\
\hline Married & 47 & $65 \cdot 3$ & 30 & $42 \cdot 3$ & 93 & $66 \cdot 9$ & \\
\hline Single & 25 & $34 \cdot 7$ & 41 & $57 \cdot 7$ & 46 & $33 \cdot 1$ & \\
\hline \multicolumn{8}{|l|}{ B. Weight-related status } \\
\hline $\mathrm{BMI}$ & & & & & & & 0.458 \\
\hline Underweight & 8 & $11 \cdot 4$ & 10 & $14 \cdot 7$ & 7 & $5 \cdot 4$ & \\
\hline Normal weight & 49 & $70 \cdot 0$ & 48 & $70 \cdot 6$ & 96 & $73 \cdot 8$ & \\
\hline Overweight & 11 & $15 \cdot 7$ & 8 & $11 \cdot 8$ & 22 & $16 \cdot 9$ & \\
\hline Obese & 2 & $2 \cdot 9$ & 2 & $2 \cdot 9$ & 5 & $3 \cdot 8$ & \\
\hline Satisfaction with physical fitness & & & & & & & 0.998 \\
\hline Satisfied & 26 & $36 \cdot 6$ & 26 & $36 \cdot 6$ & 50 & $36 \cdot 2$ & \\
\hline Unsatisfied & 45 & $63 \cdot 4$ & 45 & $63 \cdot 4$ & 88 & $63 \cdot 8$ & \\
\hline BMI \& weight perception & & & & & & & $0 \cdot 293$ \\
\hline $\mathrm{BMI}<24 \mathrm{~kg} / \mathrm{m}^{2} \&$ consider appropriate weight & 31 & $44 \cdot 9$ & 29 & $42 \cdot 6$ & 41 & $31 \cdot 5$ & \\
\hline $\mathrm{BMI}<24 \mathrm{~kg} / \mathrm{m}^{2} \&$ consider overweight & 25 & $36 \cdot 2$ & 29 & $42 \cdot 6$ & 62 & $47 \cdot 7$ & \\
\hline $\mathrm{BMI} \geq 24 \mathrm{~kg} / \mathrm{m}^{2}$ & 13 & $18 \cdot 8$ & 10 & $14 \cdot 7$ & 27 & $20 \cdot 8$ & \\
\hline \multicolumn{8}{|l|}{ C. Teaching-related status } \\
\hline Years of teaching & & & & & & & $<0.001$ \\
\hline$\leq 5$ & 17 & $24 \cdot 6$ & 51 & $75 \cdot 0$ & 26 & $19 \cdot 1$ & \\
\hline$>5$ & 52 & $75 \cdot 4$ & 17 & $25 \cdot 0$ & 110 & $80 \cdot 9$ & \\
\hline Teaching status & & & & & & & 0.386 \\
\hline Tutor & 46 & $64 \cdot 8$ & 42 & $61 \cdot 8$ & 90 & $66 \cdot 7$ & \\
\hline Non-tutor & 25 & $35 \cdot 2$ & 26 & $38 \cdot 2$ & 45 & $33 \cdot 3$ & \\
\hline Health education course instructor & & & & & & & 0.046 \\
\hline Yes & 35 & $50 \cdot 0$ & 21 & $31 \cdot 3$ & 65 & $47 \cdot 8$ & \\
\hline No & 35 & $50 \cdot 0$ & 46 & $68 \cdot 7$ & 71 & $52 \cdot 2$ & \\
\hline Participation in nutrition-related training in the past 3 years & & & & & & & $<0.001$ \\
\hline Yes & 28 & $38 \cdot 9$ & 9 & $12 \cdot 7$ & 25 & $18 \cdot 2$ & \\
\hline \multirow[t]{2}{*}{ No } & 44 & $61 \cdot 1$ & 62 & $87 \cdot 3$ & 112 & $81 \cdot \overline{8}$ & \\
\hline & Mean & SD & Mean & SD & Mean & SD & $P$ value $\S$ \\
\hline \multicolumn{8}{|l|}{ D. Scale (continuous variables) } \\
\hline Nutrition knowledge (range 0-30) & $21 \cdot 1^{a}$ & $2 \cdot 8$ & $18 \cdot 5^{\mathrm{b}}$ & $3 \cdot 0$ & $19 \cdot 1^{b}$ & $3 \cdot 4$ & $<0.001$ \\
\hline Nutrient intake behaviour (range $0-30$ ) & $22 \cdot 1^{a}$ & $3 \cdot 0$ & $20 \cdot 2^{\mathrm{b}}$ & $3 \cdot 5$ & $21 \cdot 7^{\mathrm{a}}$ & $3 \cdot 5$ & 0.001 \\
\hline Fatty food consumption (range 0-20) & $6 \cdot 6^{\mathrm{a}}$ & $1 \cdot 7$ & $6 \cdot 7^{\mathrm{b}}$ & $2 \cdot 1$ & $6 \cdot 0^{\mathrm{b}}$ & $1 \cdot 9$ & 0.026 \\
\hline Snacks and sweetened beverage consumption (range 0-15) & $5 \cdot 6$ & $2 \cdot 2$ & $5 \cdot 8$ & $1 \cdot 9$ & $5 \cdot 5$ & $2 \cdot 2$ & 0.554 \\
\hline Vegetable \& fruit consumption (range $0-15$ ) & $12 \cdot 2$ & $2 \cdot 3$ & $11 \cdot 3$ & $2 \cdot 8$ & $11 \cdot 4$ & $2 \cdot 6$ & 0.054 \\
\hline
\end{tabular}

\section{Relationship of dietary outcomes and bealth- promoting school status with weight-related status}

Table 2 shows the results of a multivariate regression model adjusting for teachers' sex, age, marital status, years of teaching, teaching status and teaching 'health education' course status. Overall, HP-D teachers scored higher on nutrition knowledge $(\beta=2 \cdot 0, P<0 \cdot 001)$ and consumption of vegetables and fruits $(\beta=1 \cdot 0, P=0 \cdot 01)$ compared with NHP teachers. We found no significant differences for nutrient intake behaviours and fatty food consumption between HP-D and NHP teachers. Among teachers in the HP-ND and NHP groups, no statistically significant differences were observed. In general, the effects of HPS status on teachers' nutrition knowledge and dietary intake were relatively more prominent than those of other sociodemographic variables. 


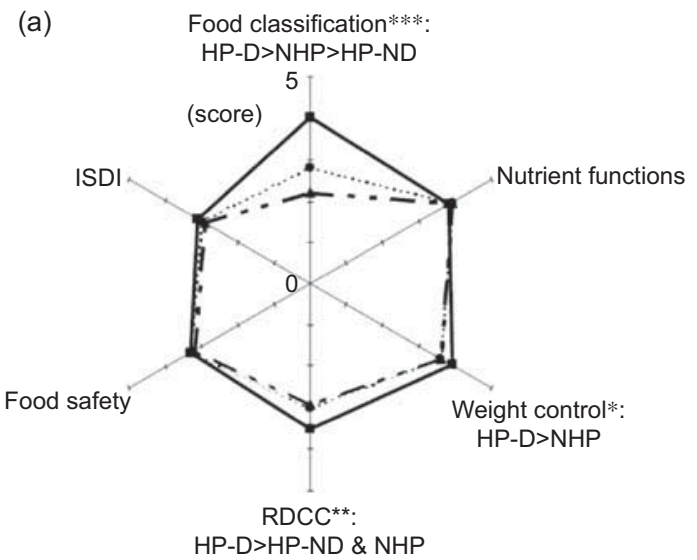

(b) Five-a-day for vegetables and fruit*: HP-D $>$ NHP $>$ HP-ND

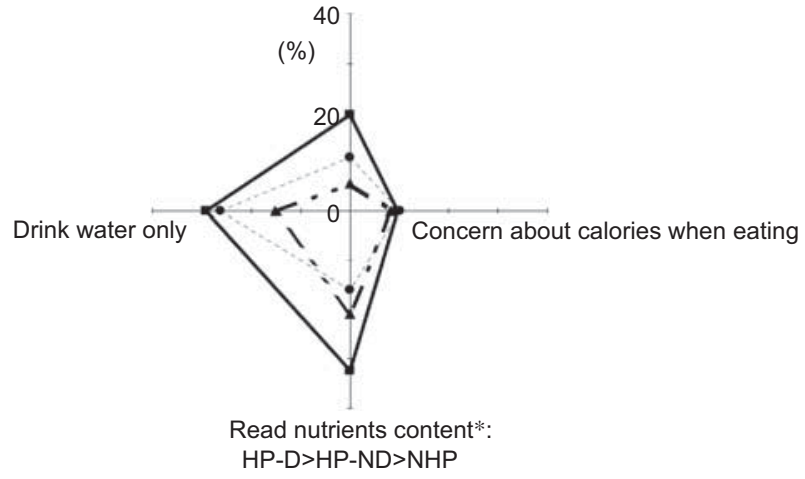

Fig. 1 (a) Distribution of scores in six categories of nutrition knowledge (ISDI, investigation on schoolchildren's dietary intake; RDCC, recommended daily consumption in childhood) and (b) proportions of participants 'always' following four particular dietary behaviours, by health-promoting school status (Taiwan, 2007): HP-D, health-promoting school aiming at dietary intervention (- - - ); HP-ND, health-promoting school not aiming at dietary intervention $(-\mathbf{\Delta}--)$; NHP, nonhealth-promoting school (- - - -). Score or proportion was significantly different among groups: ${ }^{\star} P<0.05,{ }^{\star \star} P<0.01$, ${ }^{\star \star \star} P<0.001$

Furthermore, as shown in Table 3, for those with BMI $<24 \mathrm{~kg} / \mathrm{m}^{2}$ who consider themselves to be overweight, significantly greater vegetable and fruit consumption $(\beta=1 \cdot 6, P=0 \cdot 02)$, in addition to greater nutrition knowledge $(\beta=1 \cdot 9, P=0 \cdot 02)$, was reported in the HP-D group than in the NHP group after teachers' sex, age and marital status were controlled for. HP-D teachers who were satisfied with their physical fitness had significantly higher scores on nutrition knowledge $(\beta=2 \cdot 8, P=0 \cdot 002)$, but also exhibited greater consumption of fatty food $(\beta=0 \cdot 9$, $P=0 \cdot 02$ ) compared with NHP teachers. For HP-D teachers who were unsatisfied with their physical fitness, significantly higher scores on nutrition knowledge $(\beta=1 \cdot 7$, $P=0 \cdot 01)$ and vegetable and fruit consumption $(\beta=1 \cdot 2$, $P=0 \cdot 01$ ) were found compared with unsatisfied NHP teachers. Little difference was observed between teachers in the HP-ND and NHP groups in terms of nutrition knowledge and dietary intake.

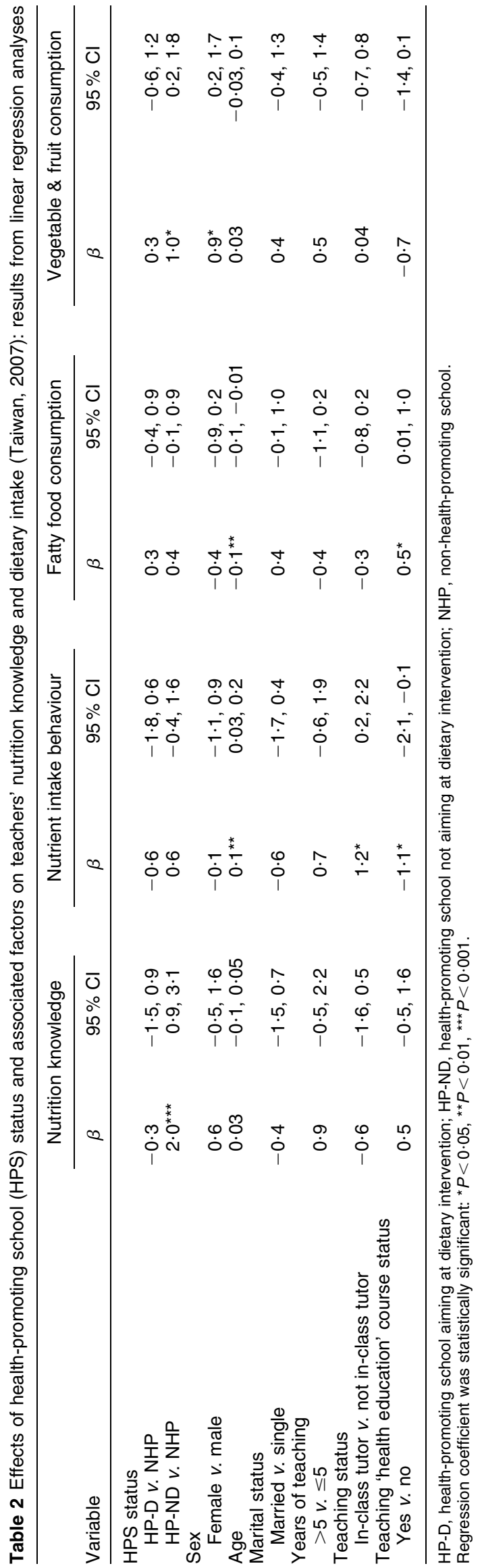


Table 3 Effects of health-promoting school status on nutrition knowledge and dietary intake by weight-related and physical fitness-related status (Taiwan, 2007): results from linear regression analyses

\begin{tabular}{|c|c|c|c|c|c|c|c|c|}
\hline \multirow[b]{2}{*}{ Scale } & \multicolumn{2}{|c|}{ Nutrition knowledge } & \multicolumn{2}{|c|}{$\begin{array}{l}\text { Nutrient intake } \\
\text { behaviour }\end{array}$} & \multicolumn{2}{|c|}{$\begin{array}{l}\text { Fatty food } \\
\text { consumption }\end{array}$} & \multicolumn{2}{|c|}{$\begin{array}{l}\text { Vegetable \& fruit } \\
\text { consumption }\end{array}$} \\
\hline & $\beta+$ & $95 \% \mathrm{Cl}$ & $\beta \dagger$ & $95 \% \mathrm{Cl}$ & $\beta+$ & $95 \% \mathrm{Cl}$ & $\beta \dagger$ & $95 \% \mathrm{Cl}$ \\
\hline \multicolumn{9}{|c|}{$\begin{array}{l}\text { A. BMI \& weight perception } \\
\mathrm{BMI}<24 \mathrm{~kg} / \mathrm{m}^{2} \& \text { consider appropriate weight }\end{array}$} \\
\hline $\begin{array}{l}\text { HP-D v. NHP } \\
\text { HP-ND v. NHP }\end{array}$ & $\begin{array}{l}1 \cdot 7^{*} \\
0 \cdot 1\end{array}$ & $\begin{array}{r}0 \cdot 0,3 \cdot 4 \\
-1 \cdot 7,1 \cdot 8\end{array}$ & $\begin{array}{l}1 \cdot 9^{*} \\
0.9\end{array}$ & $\begin{array}{r}0 \cdot 1,3 \cdot 6 \\
-0 \cdot 8,2 \cdot 7\end{array}$ & $\begin{array}{r}0.2 \\
-0.2\end{array}$ & $\begin{array}{l}-0 \cdot 8,1 \cdot 1 \\
-1 \cdot 1,0 \cdot 8\end{array}$ & $\begin{array}{l}1 \cdot 1 \\
1 \cdot 1\end{array}$ & $\begin{array}{l}-0 \cdot 2,2 \cdot 3 \\
-0 \cdot 2,2 \cdot 3\end{array}$ \\
\hline \multicolumn{9}{|c|}{$\mathrm{BMI}<24 \mathrm{~kg} / \mathrm{m}^{2} \&$ consider overweight } \\
\hline $\begin{array}{l}\text { HP-D v. NHP } \\
\text { HP-ND v. NHP }\end{array}$ & $\begin{array}{c}1 \cdot 9^{*} \\
-0 \cdot 1\end{array}$ & $\begin{array}{r}0 \cdot 3,3 \cdot 6 \\
-1 \cdot 8,1 \cdot 6\end{array}$ & $\begin{array}{r}0.5 \\
-1 \cdot 1\end{array}$ & $\begin{array}{l}-1 \cdot 1,2 \cdot 2 \\
-2 \cdot 7,0 \cdot 6\end{array}$ & $\begin{array}{l}0.6 \\
0.9\end{array}$ & $\begin{array}{r}-0 \cdot 3,1 \cdot 6 \\
0 \cdot 1,1 \cdot 8\end{array}$ & $\begin{array}{r}1 \cdot 6^{*} \\
-0 \cdot 9\end{array}$ & $\begin{array}{r}0 \cdot 3,2 \cdot 8 \\
-2 \cdot 1,0 \cdot 4\end{array}$ \\
\hline $\begin{array}{c}\text { BMI } \geq 24 \mathrm{~kg} / \mathrm{m}^{2} \\
\mathrm{HP}-\mathrm{D} v . \mathrm{NHP} \\
\mathrm{HP}-\mathrm{ND} v . \mathrm{NHP}\end{array}$ & $\begin{array}{l}3 \cdot 0^{*} \\
-2 \cdot 7\end{array}$ & $\begin{array}{r}0 \cdot 0,6 \cdot 0 \\
-5 \cdot 5,0 \cdot 1\end{array}$ & $\begin{array}{l}-0 \cdot 9 \\
-2 \cdot 8^{*}\end{array}$ & $\begin{array}{l}-3 \cdot 2,1 \cdot 4 \\
-5 \cdot 2,-0 \cdot 5\end{array}$ & $\begin{array}{r}0 \cdot 0 \\
-0 \cdot 2\end{array}$ & $\begin{array}{l}-1 \cdot 2,1 \cdot 2 \\
-1 \cdot 4,1 \cdot 0\end{array}$ & $\begin{array}{l}0 \cdot 1 \\
1 \cdot 4\end{array}$ & $\begin{array}{l}-1 \cdot 8,2 \cdot 0 \\
-0 \cdot 5,3 \cdot 4\end{array}$ \\
\hline \multicolumn{9}{|c|}{$\begin{array}{l}\text { B. Physical fitness satisfaction } \\
\text { Satisfied }\end{array}$} \\
\hline $\begin{array}{l}\text { HP-D v. NHP } \\
\text { HP-ND v. NHP }\end{array}$ & $\begin{array}{l}2 \cdot 8^{\star \star} \\
-1 \cdot 1\end{array}$ & $\begin{array}{r}1 \cdot 0,4 \cdot 6 \\
-2 \cdot 7,0 \cdot 6\end{array}$ & $\begin{array}{r}0.3 \\
-0.3\end{array}$ & $\begin{array}{l}-1 \cdot 5,2 \cdot 2 \\
-2 \cdot 0,1 \cdot 5\end{array}$ & $\begin{array}{l}0.9^{\star} \\
0.4\end{array}$ & $\begin{array}{r}0 \cdot 1,1 \cdot 7 \\
-0 \cdot 4,1 \cdot 1\end{array}$ & $\begin{array}{l}0 \cdot 6 \\
0 \cdot 4\end{array}$ & $\begin{array}{l}-0 \cdot 8,2 \cdot 0 \\
-0.9,1 \cdot 8\end{array}$ \\
\hline \multicolumn{9}{|l|}{ Unsatisfied } \\
\hline $\begin{array}{l}\text { HP-D v. NHP } \\
\text { HP-ND v. NHP }\end{array}$ & $\begin{array}{l}1 \cdot 7^{*} \\
-0.5\end{array}$ & $\begin{array}{r}0 \cdot 4,3 \cdot 1 \\
-2 \cdot 0,0 \cdot 9\end{array}$ & $\begin{array}{c}0 \cdot 8 \\
-1 \cdot 3^{*}\end{array}$ & $\begin{array}{l}-0 \cdot 4,2 \cdot 0 \\
-2 \cdot 6,0 \cdot 0\end{array}$ & $\begin{array}{l}0.2 \\
0.5\end{array}$ & $\begin{array}{l}-0 \cdot 6,1 \cdot 0 \\
-0 \cdot 3,1 \cdot 3\end{array}$ & $\begin{array}{l}1 \cdot 2^{*} \\
0 \cdot 1\end{array}$ & $\begin{array}{r}0 \cdot 3,2 \cdot 1 \\
-0 \cdot 9,1 \cdot 1\end{array}$ \\
\hline
\end{tabular}

HP-D, health-promoting school aiming at dietary intervention; HP-ND, health-promoting school not aiming at dietary intervention; NHP, non-health-promoting school. Regression coefficient was statistically significant: ${ }^{*} P<0 \cdot 05,{ }^{* \star} P<0.01$.

tControlling for sex, age and marital status.

Table 4 Effects of health-promoting school status on nutrition knowledge and dietary intake by teaching-related status (Taiwan, 2007): results from linear regression analyses

\begin{tabular}{|c|c|c|c|c|c|c|c|c|}
\hline \multirow[b]{2}{*}{ Scale } & \multicolumn{2}{|c|}{ Nutrition knowledge } & \multicolumn{2}{|c|}{$\begin{array}{l}\text { Nutrient intake } \\
\text { behaviour }\end{array}$} & \multicolumn{2}{|c|}{$\begin{array}{l}\text { Fatty food } \\
\text { consumption }\end{array}$} & \multicolumn{2}{|c|}{$\begin{array}{l}\text { Vegetable \& fruit } \\
\text { consumption }\end{array}$} \\
\hline & $\beta \dagger$ & $95 \% \mathrm{Cl}$ & $\beta \dagger$ & $95 \% \mathrm{Cl}$ & $\beta \dagger$ & $95 \% \mathrm{Cl}$ & $\beta+$ & $95 \% \mathrm{Cl}$ \\
\hline \multicolumn{9}{|c|}{$\begin{array}{l}\text { A. Years of teaching } \\
\leq 5 \text { years of teaching }\end{array}$} \\
\hline HP-D v. NHP & 1.7 & $-0 \cdot 5,4 \cdot 0$ & $1 \cdot 0$ & $-1 \cdot 3,3 \cdot 3$ & 0.0 & $-1 \cdot 5,1 \cdot 5$ & $1 \cdot 3$ & $-0.4,3.0$ \\
\hline HP-ND v. NHP & -0.7 & $-2 \cdot 5,1 \cdot 2$ & $0 \cdot 2$ & $-1 \cdot 7,2 \cdot 0$ & $-0 \cdot 1$ & $-1 \cdot 3,1 \cdot 1$ & $0 \cdot 7$ & $-0 \cdot 7,2 \cdot 0$ \\
\hline \multicolumn{9}{|c|}{$>5$ years of teaching } \\
\hline HP-D v. NHP & $2 \cdot 2^{\star \star}$ & $0.9,3.5$ & $0 \cdot 6$ & $-0 \cdot 6,1 \cdot 7$ & $0 \cdot 4$ & $-0.1,0.9$ & $1 \cdot 0^{\star}$ & $0 \cdot 1,1 \cdot 9$ \\
\hline HP-ND $v$. NHP & $-1 \cdot 0$ & $-2 \cdot 9,0 \cdot 9$ & $-1 \cdot 5$ & $-3 \cdot 3,0 \cdot 3$ & 0.5 & $-0 \cdot 3,1 \cdot 3$ & $0 \cdot 3$ & $-1 \cdot 1,1 \cdot 7$ \\
\hline \multicolumn{9}{|l|}{ B. Teaching status } \\
\hline \multicolumn{9}{|l|}{ Tutor } \\
\hline HP-D v. NHP & $2 \cdot 5^{\star \star \star}$ & $1 \cdot 2,3 \cdot 8$ & $0 \cdot 8$ & $-0 \cdot 6,2 \cdot 1$ & $0 \cdot 4$ & $-0 \cdot 3,1 \cdot 1$ & $1 \cdot 1^{*}$ & $0 \cdot 1,2 \cdot 2$ \\
\hline HP-ND v. NHP & -0.7 & $-2 \cdot 0,0 \cdot 7$ & -0.5 & $-1.9,0.9$ & $0 \cdot 2$ & $-0.5,1 \cdot 0$ & $0 \cdot 3$ & $-0 \cdot 7,1 \cdot 4$ \\
\hline \multicolumn{9}{|l|}{ Non-tutor } \\
\hline HP-D v. NHP & 0.7 & $-1 \cdot 1,2 \cdot 6$ & $0 \cdot 1$ & $-1 \cdot 6,1 \cdot 7$ & $0 \cdot 4$ & $-0 \cdot 4,1 \cdot 2$ & $0 \cdot 7$ & $-0.5,2 \cdot 0$ \\
\hline HP-ND v. NHP & -0.6 & $-2 \cdot 6,1 \cdot 3$ & $-1 \cdot 6$ & $-3 \cdot 3,0 \cdot 2$ & $0 \cdot 6$ & $-0 \cdot 2,1 \cdot 5$ & $-0 \cdot 2$ & $-1 \cdot 5,1 \cdot 2$ \\
\hline \multicolumn{9}{|c|}{ C. Teaching 'health education' course status } \\
\hline \multicolumn{9}{|c|}{ Yes } \\
\hline HP-D v. NHP & $2 \cdot 6^{\star \star \star}$ & $1 \cdot 3,3 \cdot 9$ & 0.9 & $-0 \cdot 7,2 \cdot 4$ & $0 \cdot 4$ & $-0 \cdot 5,1 \cdot 3$ & $1 \cdot 4^{*}$ & $0 \cdot 2,2 \cdot 5$ \\
\hline HP-ND v. NHP & $-1 \cdot 1$ & $-2 \cdot 7,0 \cdot 5$ & $-1 \cdot 3$ & $-3 \cdot 1,0 \cdot 5$ & $0 \cdot 2$ & $-0 \cdot 8,1 \cdot 3$ & $-0 \cdot 2$ & $-1 \cdot 6,1 \cdot 1$ \\
\hline \multicolumn{9}{|l|}{ No } \\
\hline HP-D v. NHP & $1 \cdot 6^{*}$ & $0 \cdot 0,3 \cdot 3$ & $0 \cdot 3$ & $-1 \cdot 1,1 \cdot 7$ & $0 \cdot 3$ & $-0 \cdot 4,1 \cdot 0$ & $0 \cdot 7$ & $-0 \cdot 4,1 \cdot 7$ \\
\hline HP-ND v. NHP & $-0 \cdot 1$ & $-1 \cdot 7,1 \cdot 5$ & -0.5 & $-1.9,0.9$ & $0 \cdot 6$ & $-0 \cdot 1,1 \cdot 3$ & $0 \cdot 4$ & $-0 \cdot 7,1 \cdot 5$ \\
\hline
\end{tabular}

HP-D, health-promoting school aiming at dietary intervention; HP-ND, health-promoting school not aiming at dietary intervention; NHP, non-health-promoting school. Regression coefficient was statistically significant: ${ }^{*} P<0 \cdot 05,{ }^{\star \star} P<0 \cdot 01,{ }^{\star \star \star} P<0 \cdot 001$. tControlling for sex, age, marital status and BMI.

\section{Relationship of dietary outcomes and bealth- promoting school status to teaching-related status} Finally, we performed analyses to investigate whether teaching-related status was associated with different HPS effects. Being a teacher for more than 5 years and being an in-class tutor or a 'health education' course instructor were associated with significantly higher scores on nutrition knowledge $(\beta=2 \cdot 2, \quad P=0 \cdot 001 ; \quad \beta=2 \cdot 5, \quad P<0.001 ;$ and $\beta=2 \cdot 6, P<0 \cdot 001$, respectively) and vegetable and fruit consumption $(\beta=1 \cdot 0, \quad P=0 \cdot 03 ; \quad \beta=1 \cdot 1, \quad P=0 \cdot 03$; and $\beta=1 \cdot 4, P=0 \cdot 02$, respectively) in the HP-D group compared with the NHP group (Table 4). For teachers with other 
teaching-related statuses, the differences were mostly insignificant. No significant differences were found between the HP-ND and NHP groups by various teaching-related statuses.

\section{Discussion}

The present study is the first with a particular focus on the impact of HPS implementation on schoolteachers' nutrition knowledge and dietary intake. An important finding was that HP-D teachers have significantly more nutrition knowledge than HP-ND and NHP teachers, regardless of their weight-related status. In contrast, although better nutrient intake behaviours and greater consumption of vegetables and fruits were observed for HP-D teachers, the effects were weaker and inconsistent across various behaviours. In addition, the effects of HPS implementation varied by teaching-related status, with greater influence on more senior teachers and those who were more involved with nutrition health education.

Several limitations of our study merit attention. First, the lack of a cohort pre-post design may limit evaluation of the extent of change in dietary knowledge and behaviours among schoolteachers caused by HPS implementation. In Taiwan, however, there are nine universities of education (formally named 'Normal College') that exclusively train teachers for elementary schools, with standardized curriculum in training. Almost all teachers in elementary schools are prepared uniformly in this national public education system. Thus, teachers in the HP-D, HP-ND and NHP groups are likely to be fairly homogeneous, enhancing comparability among these schools. Second, teachers may answer behavioural questions in socially preferred ways. Nevertheless, teachers, who usually identify themselves as role models for their students, may tend to exhibit similar extents of such bias. Thus, the evaluation of differences among the four schools should be relatively unaffected by this bias. The use of self-reported height and weight may contribute to measurement error, with greater discrepancies among larger people. However, self-reported data may be justifiable in a community setting such as the one used here as less than one-fifth of teachers are overweight ${ }^{(22)}$. Third, dietary behaviours were assessed using consumption frequency in the preceding week but not by precise measurement of daily intake in grams or portions. Thus, our study may have limited power to detect more subtle effects of HPS implementation on diet. Further, because the HPS-D focused on HPS 'nutrition and diet' interventions that continued for more than 2 years and involved many different activities, courses and workshops, it is difficult to separate out the effects of each HPS intervention programme and to examine how each one would independently affect teachers' nutrition knowledge and dietary habits. It is thus essential to further build a robust system to assess the process of HPS and to evaluate which areas of actions would contribute significantly to the promotion of health. Finally, the underlying concept of the HPS framework is eco-holistic. Apart from the issues specifically discussed here, the present study could not investigate potential modifying factors related to the community and the environment.

Despite these limitations, the data collected in the present study deserve further exploration. Over the last decade, the eco-holistic approach of the HPS framework has been implemented in many countries to target various health issues, including oral health, tobacco control, physical activity, and nutrition and diet ${ }^{(23-28)}$. Available studies have discussed essential elements of effective HPS implementation and have led to diverse intervention programmes that comprehensively embrace the HPS concept. Such programmes were well-integrated into the curriculum, the environment and the community, displaying encouraging outcomes in advancing health globally $^{(29-31)}$, such as in Australia ${ }^{(25,32-34)}$, Latin America $^{(35)}$, Africa ${ }^{(36-38)}$ and Europe ${ }^{(39,40)}$. With respect to Asian countries, Hong Kong initiated the Hong Kong Healthy Schools Award (HSA) in 2001 to adopt the wholeschool approach in addressing health concerns ${ }^{(41,42)}$. It was found that students in the HSA had better personal hygiene practices, knowledge of health and hygiene, access to health information, self-reported health status and academic results compared with non-health-promoting schools ${ }^{(43,44)}$, with improved school health policies, higher levels of community participation and better hygienic environments observed among HSA schools ${ }^{(43)}$. Furthermore, the success of HPS depends mainly on teachers' understanding of its building blocks ${ }^{(45)}$, which underscores the essential role of teachers.

In Taiwan, with extra funding from the Ministry of Education and increased personnel and time for the HPS implementation, the HP-D participating in the present study ran a series of health-promotion activities, including nutrition training programmes for school staff and outreach to families and communities. For example, in addition to providing more accurate information and nutritious meals, in the community outreach area, breakfast shops located near schools were encouraged to prepare healthier meals under the supervision of the school dietitian. This multilevel, multi-strategy project tried to promote a healthy diet for students, teachers and community members over two consecutive years. It is especially important in this regard to assess schoolteachers' nutrition knowledge and dietary behaviours, because teachers are key participants in HPS implementation and guiding the next generation.

The results of our study showed that the HP-D schoolteachers had better nutrition knowledge than their HP-ND and NHP counterparts. This may be due to the finding that 20-25\% more HP-D teachers than HP-ND and NHP teachers had participated in nutrition-related training in the past 3 years. Furthermore, scores on six 
categories of nutrition knowledge were consistently higher and more evenly distributed in HP-D teachers, indicating comprehensive enhancement of knowledge with HPS implementation. Similar results were reported by Shi-Chang et al., who found increases in nutrition knowledge from baseline to final evaluation for students, staff and parents ${ }^{(28)}$. Previous studies found that nutrition knowledge was unrelated to BMI ${ }^{(46,47)}$ and in our study, greater nutrition knowledge was consistently observed for HP-D teachers, regardless of weight-related status. The effects differed by teaching-related status, however, with senior teachers, in-class tutors and health education teachers having significantly better nutrition knowledge in the HP-D group than in the NHP group. This difference may be due to the greater involvement of these types of teachers in HPS implementation.

Differences in teachers' dietary behaviours were more inconsistent between the three types of schools than the differences found in nutrition knowledge. Previous studies did not find a strong association between nutrition knowledge and food intake ${ }^{(48-50)}$. Moreover, nutrition knowledge was more strongly related to fruit and vegetable consumption than to fat intake in a previous study $^{(51)}$, and our study identified similar trends. Generally, higher scores on nutrient intake behaviours were observed for HP-D teachers; however, no significant differences were found among schools in consumption frequencies of fatty food, snacks and sweetened beverages. The higher knowledge of HP-D teachers may translate better to vegetable and fruit consumption than to consumption of fatty food or snacks. Previous studies found that body image dissatisfaction or a wish to be thinner was associated with healthier food intake ${ }^{(52,53)}$. Similarly, in our study, HP-D teachers dissatisfied with their physical fitness consumed significantly more vegetables and fruits than dissatisfied NHP teachers, while among teachers who were satisfied with their physical fitness, HP-D teachers reported higher fatty food consumption than NHP teachers. As such, healthy eating patterns should be continually encouraged in teachers unsatisfied with their physical fitness and body image. In terms of teaching-related status, HP-D teachers who were more involved in HPS implementation, such as those who taught a health education course, consumed significantly more vegetables and fruits than their NHP counterparts.

Of particular concern in our findings are the inappropriate dietary behaviours displayed by some teachers, although the proportions of such teachers were higher in the HP-ND and NHP groups than in the HP-D group. For example, only $19 \cdot 4 \%$ of HP-D schoolteachers always consumed five servings of vegetables and fruits daily; with that percentage being $5 \cdot 6 \%$ and $10 \cdot 8 \%$ in the HPND and NHP groups, respectively. About one-quarter of schoolteachers in the HP-ND and NHP groups regularly skipped breakfast. Even with better nutrition education, many teachers still have inadequate diets, as reported in other studies ${ }^{(14)}$. Approximately $40 \%$ of schoolteachers (36.2\% in the HP-D group, $42 \cdot 7 \%$ in the HP-ND group and $47.7 \%$ in the NHP group) who had normal BMI status $\left(\mathrm{BMI}<24 \mathrm{~kg} / \mathrm{m}^{2}\right)$ considered themselves to be overweight, exemplifying the cultural stereotype of thinness as an ideal. These unhealthy attitudes and behaviours may be inadvertently transferred to students through classroom education or role modelling ${ }^{(14)}$.

Finally, our study identified significant effects of HPS implementation only on the HP-D schoolteachers. The HPND and NHP groups exhibited similar patterns of insignificant differences from the HP-D group. Although HPS implementation stresses an eco-holistic approach, with comprehensive health promotion efforts, there are difficulties and complexities in practice due to restricted budgets, personnel and time. In Taiwan, HPS were encouraged to select a special intervention as a first priority, which was found to be a feasible and practical strategy.

Future research would benefit from a broader range of evaluation strategies to examine the implementation of HPS more comprehensively. Such research should address whether embracing the concept of HPS or executing specific intervention programmes makes the change, and how HPS implementation affects students, teachers and community members. As suggested by Worsley ${ }^{(54)}$, follow-up studies are needed to continue monitoring and evaluating the long-term effects of HPS implementation on teachers as well as on students and community members.

\section{Conclusion}

Overall, the results of our study indicate that HPS implementation with a focus on nutrition and diet correlates positively with schoolteachers' health. Improvement of schoolteachers' health knowledge and behaviours should be considered an ongoing process from both personal and professional perspectives. With concurrent incorporation of both classroom education and changes in school policy, environment, services and community outreach, the coordinated HPS framework can be further expanded.

\section{Acknowledgements}

This research was supported by a grant from the National Health Research Institute, Taiwan (DOH94-TD-M-113071-(2)), the Ministry of Education, Taiwan and the Bureau of Health Promotion, Department of Health, Taiwan. There is no conflict of interest. Y.-H.C. designed the study, analysed the data and wrote the paper. C.-Y.Y. conceived the strategies and supervised the project. Y.-M.L. carried out the field investigation and collected the data. M.-L.H. and K.-C.H. assisted in analysing and interpreting the data. H.-Y.C. designed the study and 
supervised the project. The authors are grateful for the valuable contributions of all of the teachers and staff in the four participating schools, as well as four key consultants, Han-Wen Yen, Chieh-Hsing Liu, J.L. Guo and S.C. Yang.

\section{References}

1. Moore VM \& Davies MJ (2005) Diet during pregnancy, neonatal outcomes and later health. Reprod Fertil Dev 17, 341-348.

2. Wright CM, Parker L, Lamont D \& Craft AW (2001) Implications of childhood obesity for adult health: findings from Thousand Families Cohort Study. BMJ 323, 1280-1284.

3. Adams LB (1997) An overview of adolescent eating behavior barriers to implementing dietary guidelines. Ann N Y Acad Sci 817, 36-48.

4. Casagrande SS, Wang Y, Anderson C \& Gary TL (2007) Have Americans increased their fruit and vegetable intake? The trends between 1988 and 2002. Am J Prev Med 32, 257-263.

5. Department of Health (2006) Reports from the Nutritional Status of the Elderly and School Children in the Second Nutritional and Health Survey in Taiwan. Taiwan: Bureau of Health Promotion, Department of Health, Executive Yuan.

6. Janssen I, Katzmarzyk PT, Boyce WF, Vereecken C, Mulvihill C, Roberts C, Currie C \& Pickett W; Health Behaviour in School-Aged Children Obesity Working Group (2005) Comparison of overweight and obesity prevalence in school-aged youth from 34 countries and their relationships with physical activity and dietary patterns. Obes Rev 6, 123-132.

7. Starkey LJ, Johnson-Down L \& Gray-Donald K (2001) Food habits of Canadians: comparison of intakes in adults and adolescents to Canada's food guide to healthy eating. Can J Diet Pract Res 62, 61-69.

8. Baranowski T, Davis M, Resnicow K, Baranowski J, Doyle C, Lin LS, Smith M \& Wang DT (2000) Gimme 5 fruit, juice, and vegetables for fun and health: outcome evaluation. Health Educ Behav 27, 96-111.

9. Prell HC, Berg MC, Jonsson LM \& Lissner L (2005) A schoolbased intervention to promote dietary change. $J$ Adolesc Health 36, 529.

10. Von Post-Skagegard M, Samuelson G, Karlstrom B, Mohsen R, Berglund L \& Bratteby LE (2002) Changes in food habits in healthy Swedish adolescents during the transition from adolescence to adulthood. Eur J Clin Nutr 56, 532-538.

11. Alnasir FA \& Skerman JH (2004) Schoolteachers' knowledge of common health problems in Bahrain. East Mediterr Health J 10, 537-546.

12. Cristina PG (2006) Nutrition education in public elementary schools of Sao Paulo, Brazil: the Reducing Risks of Illness and Death in Adulthood project. Rev Nutr Campinas 19, 309-320.

13. Kubik MY, Lytle LA, Hannan PJ, Story M \& Perry CL (2002) Food-related beliefs, eating behavior, and classroom food practices of middle school teachers. J Sch Health 72, 339-345.

14. O'Dea JA \& Abraham S (2001) Knowledge, beliefs, attitudes, and behaviors related to weight control, eating disorders, and body image in Australian trainee home economics and physical education teachers. J Nutr Educ 33, 332-340.

15. Lynagh M, Schofield MJ \& Sanson-Fisher RW (1997) School health promotion programs over the past decade: a review of the smoking, alcohol and solar protection literature. Health Promot Int 12, 43-60.
16. Nutbeam D (1995) Exposing the myth. What schools can and cannot do to prevent tobacco use by young people. Promot Educ 2, 11-14., 57.

17. Macdonald G \& Bunton R (1992) Health Promotion: Disciplines and Diversity? London: Routledge.

18. World Health Organization (1986) The Ottawa Charter for Health Promotion. Ottawa: WHO Regional Office.

19. Levinger B (1996) Nutrition, Health and Education For All. Newton and New York: Education Development Center, Inc. and United Nations Development Programme.

20. Pollitt E (1990) Malnutrition and Infection in the Classroom. Paris: UNESCO.

21. Wardle J, Haase AM \& Steptoe A (2006) Body image and weight control in young adults: international comparisons in university students from 22 countries. Int J Obes (Lond) 30, 644-651.

22. Leikas S, Lindeman M, Roininen K \& Lahteenmaki L (2007) Food risk perceptions, gender, and individual differences in avoidance and approach motivation, intuitive and analytic thinking styles, and anxiety. Appetite 48, 232-240.

23. Anderson AS, Porteous LE, Foster E, Higgins C, Stead M, Hetherington M, Ha MA \& Adamson AJ (2005) The impact of a school-based nutrition education intervention on dietary intake and cognitive and attitudinal variables relating to fruits and vegetables. Public Health Nutr 8 , 650-656.

24. Moyses ST, Moyses SJ, Watt RG \& Sheiham A (2003) Associations between health promoting schools' policies and indicators of oral health in Brazil. Health Promot Int 18, 209-218.

25. Schofield MJ, Lynagh M \& Mishra G (2003) Evaluation of a Health Promoting Schools program to reduce smoking in Australian secondary schools. Health Educ Res 18, 678-692.

26. Sobczyk W, Hazel N, Reed CD, Ciarroccki B, Cohen S \& Varga D (1995) Health Promotion Schools of Excellence: a model program for Kentucky and the nation. $J$ Ky Med Assoc 93, 142-147.

27. Turunen H, Tossavainen K, Jakonen S, Salomaki U \& Vertio H (1999) Initial results from the European Network of Health Promoting Schools program on development of health education in Finland. J Sch Health 69, 387-391.

28. Shi-Chang X, Xin-Wei Z, Shui-Yang X, Shu-Ming T, Sen-Hai Y, Aldinger C \& Glasauer P (2004) Creating healthpromoting schools in China with a focus on nutrition. Health Promot Int 19, 409-418.

29. McQueen D \& Jones C (2007) Global Perspectives on Health Promotion Effectiveness. New York: Springer Science and Business Media, LLC.

30. St Leger L, Kobe L, Lee A, McCall D \& Young I (2007) School health promotion: achievements, challenges and priorities. In Global Perspective on Health Promotion Effectiveness, pp. 107-124 [D McQueen and C Jones, editors]. New York: Springer Science and Business Media, LLC.

31. Stewart-Brown S (2006) What is the Evidence on School Health Promotion in Improving School Health or Preventing Disease and Specifically What is the Effectiveness of the Health Promoting Schools Approach? Copenhagen: WHO Regional Office for Europe.

32. Marshall BJ, Sheehan MM, Northfield JR, Maher S, Carlisle R \& Leger LH (2000) School-based health promotion across Australia. J Sch Health 70, 251-252.

33. Patton GC, Bond L, Carlin JB, Thomas L, Butler H, Glover S, Catalano R \& Bowes G (2006) Promoting social inclusion in schools: a group-randomized trial of effects on student health risk behavior and well-being. Am J Public Health 96, 1582-1587.

34. Lin V \& Fawkes S (2007) Health promotion in Australia: twenty years on from the Ottawa Charter. Promot Educ 14, 203-208. 
35. Ippolito-Shepherd J, Cerqueira MT \& Ortega DP (2005) Health-Promoting Schools Regional Initiative of the Americas. Promot Educ 12, 220-229, 180.

36. Swart D \& Reddy P (1999) Establishing networks for health promoting schools in South Africa. J Sch Health 69, 47-50.

37. Mathee A \& Byrne J (1996) Towards environment and health promoting South African schools. Urban Health Newsl, 48-52.

38. Taylor M, Coovadia HM, Kvalsvig JD, Jinabhai CC \& Reddy P (1999) Helminth control as an entry point for healthpromoting schools in KwaZulu-Natal. $S$ Afr Med $J$ 89, 273-279.

39. Inchley J, Muldoon J \& Currie C (2007) Becoming a health promoting school: evaluating the process of effective implementation in Scotland. Health Promot Int 22, 65-71.

40. Barnekow Rasmussen V (2005) The European Network of Health Promoting schools - from Iceland to Kyrgyzstan. Promot Educ 12, 169-172.

41. Lee A, Cheng FF, Yuen H, Ho M, Lo A, Fung Y \& Leung T (2007) Achieving good standards in health promoting schools: preliminary analysis one year after the implementation of the Hong Kong Healthy Schools Award scheme. Public Health 121, 752-760.

42. Lee A, St Leger L \& Moon A (2005) Evaluating Health Promotion in Schools meeting the needs for education and health professionals: a case study of developing appropriate indictors and data collection methods in Hong Kong. Promot Educ 12, 123-130.

43. Lee A, Wong MC, Keung VM, Yuen HS, Cheng F \& Mok JS (2008) Can the concept of Health Promoting Schools help to improve students' health knowledge and practices to combat the challenge of communicable diseases: case study in Hong Kong? BMC Public Health 8, 42.
44. Lee A, Cheng FF, Fung Y \& St Leger L (2006) Can Health Promoting Schools contribute to the better health and wellbeing of young people? The Hong Kong experience. J Epidemiol Community Health 60, 530-536.

45. Lee A, St Leger L \& Cheng FF (2007) The status of healthpromoting schools in Hong Kong and implications for further development. Health Promot Int 22, 316-326.

46. O'Brien G \& Davies M (2007) Nutrition knowledge and body mass index. Health Educ Res 22, 571-575.

47. Thakur N \& D'Amico F (1999) Relationship of nutrition knowledge and obesity in adolescence. Fam Med 31, 122-127.

48. Pirouznia M (2000) The correlation between nutrition knowledge and eating behavior in an American school: the role of ethnicity. Nutr Health 14, 89-107.

49. Shepherd R \& Stockley L (1987) Nutrition knowledge, attitudes, and fat consumption. J Am Diet Assoc 87, 615-619.

50. Stafleu A, Van Staveren WA, De Graaf C, Burema J \& Hautvast JG (1996) Nutrition knowledge and attitudes towards high-fat foods and low-fat alternatives in three generations of women. Eur J Clin Nutr 50, 33-41.

51. Wardle J, Parmenter K \& Waller J (2000) Nutrition knowledge and food intake. Appetite 34, 269-275.

52. Contento IR, Basch C \& Zybert P (2003) Body image, weight, and food choices of Latina women and their young children. J Nutr Educ Behav 35, 236-248.

53. Macdonald LA, Wearring GA \& Moase O (1983) Factors affecting the dietary quality of adolescent girls. J Am Diet Assoc 82, 260-263.

54. Worsley A (2002) Nutrition knowledge and food consumption: can nutrition knowledge change food behaviour? Asia Pac J Clin Nutr 11, Suppl. 3, S579-S585. 